\title{
Original Article \\ Application of computed aided detection in breast masses diagnosis
}

\author{
Changizi V, Giti $\mathbf{M}^{1}$, Arab Kheradmand $\mathbf{A}^{2}$ \\ Technology of Radiology and Radiotherapy Department, Allied Health Sciences School, Tehran \\ University of Medical Sciences, Tehran, Iran. ${ }^{1}$ Radiology Department, Center of Medical Imaging, Imam \\ Khomaini Hospital, School of Medicine, Tehran University of Medical Sciences, Tehran, Iran. \\ ${ }^{2}$ Meraj Award, Imam Khomaini Hospital, School of Medicine, Tehran University of Medical Sciences, \\ Tehran, Iran.
}

Correspondence to: Dr. Vahid Changizi, E-mail: changizi@sina.tums.ac.ir

\begin{abstract}
Breast cancer is the most widespread cancer in women. The life-time risk of a woman developing this disease has been established as one in eight. Currently mammography is a standard method and could decrease breast cancer mortality. Unfortunately, negative mammograms don't exclude cancer. The sensitivity of mammography ranges from approximately $70 \%$ to $90 \%$ and it should be higher. MATERIALS AND METHODS: The sample contained 255 cases taken from Imaging Center of Imam Khomaini Hospital. Bilateral mammograms in both craniocaudal and mediolateral oblique projections were used. Two experienced Radiologists reviewed images before and after using CAD system. Tumors (including malignant and benign) and normal breast tissues were confirmed by histological correlation. RESULTS: Of 255 cases 92 were not recommended for further work-up. Of 163 cases 90 were normal mass, 23 malignant tumors, 16 benign tumors and 22 cysts were detected by CAD system. The remaining cases were finalized only by biopsy. CONCLUSION: CAD could be utilized for breast mass detection. This is a practical technique with low cost.
\end{abstract}

Key words: Breast masses, computed aided detection, mammography

\section{Introduction}

Breast cancer is the most widespread cancer in women. Breast cancer is the most frequent cancer in women in the world. It is a major cause of death in women of 33-55 years, where there has been an increase in the mortality rate in the last two decades. ${ }^{[1]}$ The lifetime risk of a woman developing this disease has been established as one in eight. ${ }^{[2]} \mathrm{M}$ ammography has been successful at reducing breast cancer mortality. ${ }^{[3]} \mathrm{A} n$ evaluation of eight mammography screening trials by the U nited States Preventive Services Task Force showed that mammography reduces breast cancer mortality by $16 \% \cdot{ }^{[4]}$ Regardless of this advantage, the false negative rate in screening mammography remains unacceptably high. ${ }^{[5,6]}$ The sensitivity of mammography ranges from approximately $70 \%$ to $90 \% .{ }^{[7]} \mathrm{U}$ nfortunately, negative mammograms don't exclude cancer. As a matter of fact improvement in mammography sensitivity is needed. Finding what makes a false negative mammogram can't be easy as months may pass before the cancer is detected.
H owever radiologists have an extremely important role since a cancer can be missed by the interpreting radiologist or because the radiologist misinterprets the finding. Clinical studies have shown that 30\%-70\% of breast cancer diagnosed at screening mammography are visible in retrospect on prior examinations and that detection errors are responsible for approximately half of missed breast cancers, with interpretation errors accounting for the other half. ${ }^{[2,8]}$ Computer methods and programs in medicine are dedicated to scientific development. The great advantage of digital image matrices is the ability to manipulate the raw image data using computer techniques. On this basis computer aided detection (CAD) for mammography is gaining clinical acceptance. In recent years, CAD has been applied to help radiologists in detecting breast cancer. In the CAD system first of all a digital mammogram is acquired. A digital image is a string of binary numbers $(0$ and 1$)$. Then the digital image is manipulated and analyzed by computer under the radiologist control. Brightness, gray scale, segmentation and edge detection are main factors 
that can be manipulated. Finally the radiologist makes the interpretation. Brem et al, reported breast cancer detection is improved with the use of computer aided detection. ${ }^{[2]}$ Freer et al, showed that the use of CAD in the interpretation of screening mammograms can increase the detection of early stage malignancies..$^{[5]} \mathrm{M}$ arx et al, reported that CAD could reduce the rate of unnecessary biopsies. ${ }^{[9]}$ Beam et al, reported that double reading of mammograms can increase breast cancer detection. ${ }^{[10]}$

The purpose of our study was to evaluate changes in tumor detection with and without the introduction of a CAD system.

\section{Materials and Methods}

The sample contained 255 cases taken from Imaging Center of Imam Khomaini Hospital. Bilateral mammograms in both craniocaudal and mediolateral oblique projections were used. Tumors (including malignant and benign) and normal breast tissues were confirmed by histological process. U sing a scanner (3200 × $1600 \mathrm{dpi}, 48$ bit color depth) images were digitized. Before any computerized manipulation, images were reported by the radiologist under blinded technique (no information of patient file). After interpretation image quality improvement abilities of software were done on images. Besides changing in brightness using gray scale and cropping two important parameters were applied for images listed below:

Segmentation: Separating an image into regions of similar attributes. Segmentation can help determine the boundary of masses.

Edge detection: To mark the points in a digital image at which the luminous intensity changes sharply. Edge detection is a research field within image processing and computer vision, in particular within the area of feature extraction. Edge detection is very important in image analysis where an edge is the change in gray levels between two objects. Both of edge detection and segmentation were applied for differentiation among normal tissue, cyst, malignant and benign tumors. Though changing brightness was used to look in better condition.

The output of the CAD system was displayed on a LCD monitor (Sony, 14.1", WXGA: 1280 × 800).

\section{Results}

Of 255 cases 92 were not recommended for further work-up. These cases included 60 normal, 20 cyst and 12 normal dense breast tissues. On the other hand
CAD had no effect on radiologist decision. CAD could help to make a decision for 159 cases including 90 normal masses, 29 malignant tumors, 17 benign tumors and 23 cysts. The rest of cases were finalized only by biopsy. Beside edge detection and segmentation some other abilities written in software could help with the interpretation. Ability to crop, selecting the brightness and embossing were three important types of them. $U$ sing this CAD system sensitivity of $92 \%$ was obtained for detecting tumors. Also it was found that this system could make differentiation between malignant and benign breast tumors. The above numbers for tumoral groups reveal this ability.

Figures 1 and 2 show malignant tumors by CAD system. Also Figure 3 shows the edge detection facility of breast tissue.

\section{Discussion}

Similar to Brem et al, M arx et al, results this study showed using computed aided detection could improve breast cancer detection. Therefore false negative rate decreased. The software written for this study was on the gray level base. On the other side breast tumors in

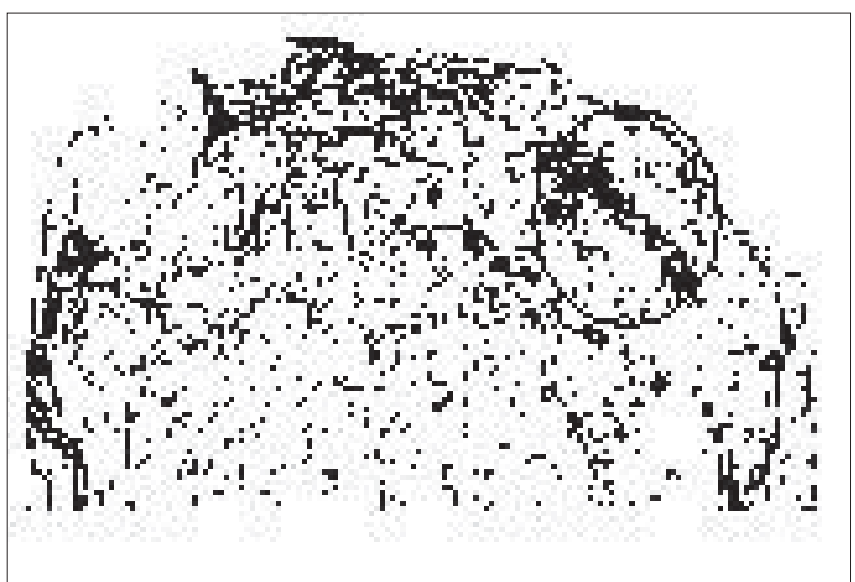

Figure 1: Using edge detection for showing malignant tumor. Mulitple microcalcification has been signed

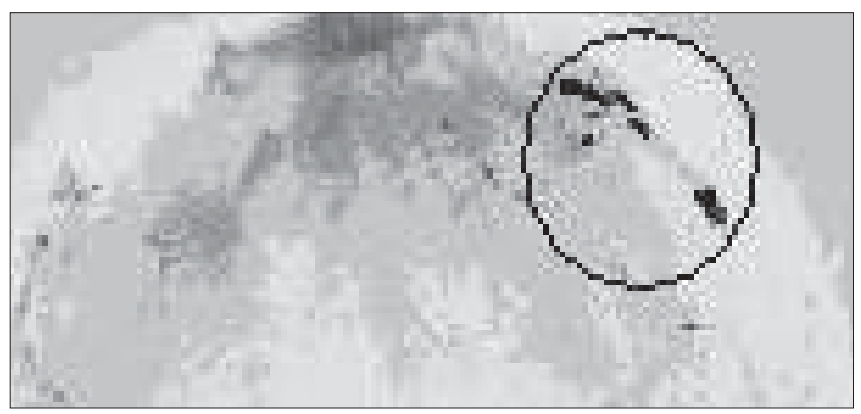

Figure 2: Using segmentation for showing malignant tumor. Mulitple microcalcification has been signed 


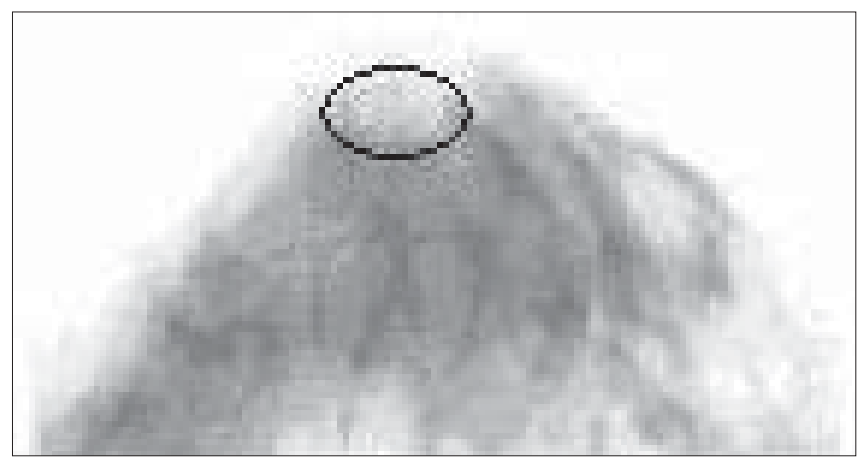

Figure 3: Using segmentation for showing benign tumor with defined boundary

a mammogram show different textures and gray levels other than the normal ones. Therefore it was possible to differentiate them from the background using image segmentation. Even though a large number of features are required for distinction between lesion and normal tissues and the radiologist can't track all of them, using a variety of edge detection levels considered in written software could help to find considerable more features. Benign and malignant breast tissues could be differentiated from each other by the capability of segmentation and edge detection. As a matter of fact a combination of parameters even brightness variation could help for interpretation. According to reports by Beam et al, double reading of mammograms can increase breast cancer detection. This practice is impractical in many centers so it couldn't gain widespread acceptance. This technique was easier and of lower cost than recall that is used in some mammography screenings.

\section{Conclusion}

This study showed CAD could decrease false negative rate. Using CAD, it was possible to differentiate between benign and malignant breast tumors. Finally this method was easy to use and of low cost than recall that is used in mammography centers. R ecall means to get another radiography that causes double charge.

\section{Acknowledgment}

This study has been supported by Tehran U niversity of Medical Sciences

\section{References}

1. Kidane G, Speller RD, Royle GJ, Hanby AM. X-ray scatter signatures for normal and neoplastic breast tissues. Phys Med Biol 1999;44:1791-802.

2. Brem RF, Baum J, Lechner M, Kaplan S, Souders S, Naul LG, et al. Improvement in sensitivity of screening mammography with computer aided detection: A multiinstitutional trial. AJR Am J Roentgenol 2003; 181:687-93.

3. Mushlim Al, Kouides RW, Shapiro DE. Estimating the accuracy of screening mammography: A meta analysis. Am J Prev Med 1998; 14:143-53.

4. Humphrey LL, Helfand M, Chan BK, Woolf SH. Breast cancer screening: A summary of the evidence for the US Preventive Services Task Force. Ann Intern Med 2002; 137:347-60.

5. Freer TW, Ulissey MJ. Screening mammography with computer aided detection: Prospective study of 12860 patients in a community breast center. Radiology 2001;220:781-6.

6. Warren Burhenne LJ, Wood SA, D' Orsi CJ, Feig SA, Kopans DB, O' Shaughnessy, et al. The potential contribution of computer aided detection to the sensitivity of screening mammography. Radiology 2000;215:554-62.

7. Yankaskas BC, Cleveland RJ, Schell MJ, Kozar R. Association of recall rates with sensitivity and positive predictive values of screening mammography. AJR Am J Roentgenol 2001;133:855-63.

8. Birdwell RL, Ikeda DM, O'Shaughnessy KF, Sickles EA. Mammography characteristic of 115 missed cancers later detected with screening mammography and the potential utility of computer aided detection. Radiology 2001;219:192-202.

9. Marx C, Malich A, Facius M, Grebenstein U, Sauner D, Pfleiderer S, et al. Are unnecessary fallow up procedures induced by computer aided diagnosis (CAD) in mammography? Comparison of mammographic diagnosis with and without use of CAD. Eur J Radiol 2004;51:66-72.

10. Beam C, Hendrick RE. Proposition: All mammograms should be double-read. Med Phys 1999;26:115-8.

Source of Support: Nil, Conflict of Interest: None declared. 